\title{
Transfer Green's functions in two-fold interaction systems
}

\author{
U Schröter and E Scheer \\ University of Konstanz, FB Physik, Universitätsstraße 10, 78464 Konstanz, Germany \\ E-mail: Ursula Schroeter@uni-konstanz.de
}

\begin{abstract}
For systems governed by two kinds of interactions it is shown that these can be built successively into the Green's functions describing the system's response. Whereas for the ordinary Green's function the Dyson equation to solve has the same form in each step, we derive the non-trivial second-step equation for the transfer or coupling function, which on the one hand is closely related to the self-energy and on the other hand of practical relevance in transport calculations.
\end{abstract}

PACS numbers: $02.60 . \mathrm{Cb}, 02.90 .+\mathrm{p}, 72.10 . \mathrm{Bg}$

\section{Introduction}

The Green's functions [1] present a powerful tool to calculate the response of complex systems, as an evolution in time or the effect of a stimulus at any location in space. Electromagnetics methods mostly aim at solving boundary value problems [2], however, evaluations of field distributions and optical densities of states in open systems [3] dominated by multiple scattering rely on the same propagators. In quantum mechanics [4-6] Green's functions are used in the context of getting density matrices that differ from those of an original system through time evolution, added potentials or interactions. Spectral functions signal resonances or collective excitations or help to calculate scattering cross sections. Transport problems present another important area of employment for Green's functions [7, 8].

The properties and recipes we discuss in the following are independent of the field of application. Nevertheless, the general derivations have been initiated by working on specific problems in electrodynamics [9] and quantum transport [10], of which the latter shall be referred to as an example. It shall be shown that several interactions or perturbations that may be of the same or of different kind, without ranking in importance or strength can be built into the Green's functions successively. This approach also works with renormalized transfer functions, interactions or susceptibilities. However, the Dyson equation for these in the second

Konstanzer Online-Publikations-System (KOPS) 
step cannot simply be guessed in analogy to the ordinary one. The stepwise construction of the Green's functions requires no approximation and thus leads to the exact results. Sections 2-6 present the general formalism, the central part being the derivation of the second-step transfer function Dyson equation in section 4. Section 7 is about our application in modeling quantum transport through a double junction.

\section{Two perturbations}

The starting point is a homogeneous differential equation

$$
D_{\xi} \phi_{0}(\xi)=0
$$

with a differential operator $D_{\xi}$ and a function $\phi_{0}(\xi)$ as the solution. In quantum mechanics (1) typically is the Schrödinger equation with $D_{\xi}$ being the Hamilton operator and $\phi_{0}$ the wavefunction. We assume that the solution $\phi_{0}$ of (1) is known. The corresponding Green's function $g$ of this unperturbed system satisfies

$$
D_{\xi} g\left(\xi, \xi^{\prime}\right)=\delta\left(\xi-\xi^{\prime}\right) .
$$

$g$ is also assumed to be known. $g$ contains even more information about the system than a special state $\phi_{0}$, because it gives the response to an elementary excitation. Now introducing a potential, scattering or coupling term $v_{1}$, the equations for the perturbed system read as

$$
D_{\xi} \phi(\xi)-v_{1}(\xi) \phi(\xi)=0
$$

for now the unknown solution $\phi$ and

$$
D_{\xi} G_{1}\left(\xi, \xi^{\prime}\right)-v_{1}(\xi) G_{1}\left(\xi, \xi^{\prime}\right)=\delta\left(\xi-\xi^{\prime}\right)
$$

for the unknown Green's function $G_{1}$ characterizing the response properties. Although additional terms to the known basic system are commonly called perturbations, in contrast to perturbation theory the Green's function technique does not restrict these additions to small changes. From (2) and (3) it can be deduced [11] that an integral equation for $G_{1}$ is

$$
G_{1}\left(\xi, \xi^{\prime}\right)=g\left(\xi, \xi^{\prime}\right)+\int \mathrm{d} \xi^{\prime \prime} g\left(\xi, \xi^{\prime \prime}\right) v_{1}\left(\xi^{\prime \prime}\right) G_{1}\left(\xi^{\prime \prime}, \xi^{\prime}\right)
$$

or

$$
G_{1}=g+g v_{1} G_{1}
$$

for short. (5) is known as the Dyson equation.

If the perturbation consists of two parts $v_{1}$ and $v_{2}$, for the complete system's Green's function $G_{2}$ we should, of course, have set up the differential equation to satisfy as

$$
D_{\xi} G_{2}\left(\xi, \xi^{\prime}\right)-\left(v_{1}(\xi)+v_{2}(\xi)\right) G_{2}\left(\xi, \xi^{\prime}\right)=\delta\left(\xi-\xi^{\prime}\right) .
$$

However, herein terms can be grouped differently and

$$
\left(D_{\xi}-v_{1}(\xi)\right) G_{2}\left(\xi, \xi^{\prime}\right)-v_{2}(\xi) G_{2}\left(\xi, \xi^{\prime}\right)=\delta\left(\xi-\xi^{\prime}\right)
$$

can be compared to (3) with $D_{\xi}-v_{1}$ instead of the original $D_{\xi}, v_{2}$ instead of $v_{1}, G_{2}$ replacing $G_{1}$ and $G_{1}$ replacing $g$. Since $g$ described the background system when building up $G_{1}$, now the system with $v_{1}$ already included and described by $G_{1}$ provides the basis for constructing $G_{2}$. Therefore

$$
G_{2}=G_{1}+G_{1} v_{2} G_{2}
$$


which represents a short notation for a contraction by integration like in (5). By inserting it and using (4), one indeed verifies that the function $G_{2}$ implicitly defined by (9) fulfils (7):

$$
\begin{aligned}
D_{\xi} G_{1}\left(\xi, \xi^{\prime}\right)+ & D_{\xi} \int \mathrm{d} \xi^{\prime \prime} G_{1}\left(\xi, \xi^{\prime \prime}\right) v_{2}\left(\xi^{\prime \prime}\right) G_{2}\left(\xi^{\prime \prime}, \xi^{\prime}\right) \\
& -v_{1}(\xi) G_{1}\left(\xi, \xi^{\prime}\right)-\int \mathrm{d} \xi^{\prime \prime} v_{1}(\xi) G_{1}\left(\xi, \xi^{\prime \prime}\right) v_{2}\left(\xi^{\prime \prime}\right) G_{2}\left(\xi^{\prime \prime}, \xi^{\prime}\right) \\
& -v_{2}(\xi) G_{1}\left(\xi, \xi^{\prime}\right)-\int \mathrm{d} \xi^{\prime \prime} v_{2}(\xi) G_{1}\left(\xi, \xi^{\prime \prime}\right) v_{2}\left(\xi^{\prime \prime}\right) G_{2}\left(\xi^{\prime \prime}, \xi^{\prime}\right) \\
= & \left(D_{\xi}-v_{1}(\xi)\right) G_{1}\left(\xi, \xi^{\prime}\right) \\
& +\int \mathrm{d} \xi^{\prime \prime} \delta\left(\xi-\xi^{\prime \prime}\right) v_{2}\left(\xi^{\prime \prime}\right) G_{2}\left(\xi^{\prime \prime}, \xi^{\prime}\right)-v_{2}(\xi) G_{2}\left(\xi, \xi^{\prime}\right) \\
= & \delta\left(\xi-\xi^{\prime}\right) .
\end{aligned}
$$

The construction of $G_{2}$ successively by solving first (6) and then (9) is equivalent to directly solving (7).

\section{Transfer Green's functions}

From (4) on terms of the form $v(\xi) G\left(\xi, \xi^{\prime}\right)$ appear. This means that an excitation is propagated from $\xi^{\prime}$ to $\xi$ with all influences from the perturbed system to maybe even repeatedly encounter the perturbation $v$ at $\xi$. For effects at $\xi$ or those further propagated all information required is the excitation at $\xi$. A renormalized perturbation function $V\left(\xi, \xi^{\prime}\right)$ is needed to describe the response at $\xi$ of the system with all perturbations, including those located elsewhere from $\xi$, if otherwise one wants to use $g\left(\xi, \xi^{\prime}\right)$ as if the excitation propagated through the unperturbed background system. Rather than renormalized perturbation we shall call $V$ the transfer Green's function for reasons explained later in the context of quantum transport calculations.

$$
V g=v G
$$

should be understood as the defining equation for $V$. Like the complementary form $G=g+G v g$ of the Dyson equation

$$
G=g+g v G
$$

(11) has the corollar with permuted factors $g V=G v$. (11) and its corollar are now used for conversion between $G$ and $V$, as $g$ and $v$ are always known. The following little manipulation translates the Dyson equation (12) for $G$ into a Dyson equation for $V$ : multiply it by $v$ from the right, then use the conversion and finally skip $g$ on the left of all terms.

$G v=g v+g v G v \Rightarrow g V=g v+g v g V \quad \Rightarrow \quad V=v+v g V$

or $V\left(\xi, \xi^{\prime}\right)=\delta\left(\xi-\xi^{\prime}\right) v(\xi)+\int \mathrm{d} \xi^{\prime \prime} v(\xi) g\left(\xi, \xi^{\prime \prime}\right) V\left(\xi^{\prime \prime}, \xi^{\prime}\right)$. (Even with products meaning integration, transformations as done to obtain (13) follow the rules as for matrix algebra.) Written in short notation, (13) is of the same structure as (12) with $g$ and $v$ as well as $G$ and $V$ interchanged.

\section{The second-step transfer function}

Just adding indices 1 in the notation, in the last section the transfer function and its Dyson equation for the system with one perturbation were obtained, that is $V_{1}=v_{1}+v_{1} g V_{1}$ in equivalence to $G_{1}=g+g v_{1} G_{1}$. Let us now introduce the second perturbation and-in 
analogy to $G_{2}$ —call the transfer function taking account of both $V_{2}$. Had the perturbations been put in at once together, we would have to work with the Dyson equations

$$
\begin{aligned}
& G_{2}=g+g\left(v_{1}+v_{2}\right) G_{2} \\
& V_{2}=\left(v_{1}+v_{2}\right)+\left(v_{1}+v_{2}\right) g V_{2}
\end{aligned}
$$

or their complementary forms. However, we here suppose that $G_{1}$ and $V_{1}$ are already calculated and using these we want to establish a Dyson equation for $V_{2}$ starting from (9). The deduction runs as follows:

$$
\begin{aligned}
& G_{2}\left(v_{1}+v_{2}\right)=G_{1}\left(v_{1}+v_{2}\right)+G_{1} v_{2} G_{2}\left(v_{1}+v_{2}\right) \\
& G_{2}\left(v_{1}+v_{2}\right)=G_{1} v_{1}+g v_{2}+g v_{1} G_{1} v_{2}+g v_{2} G_{2}\left(v_{1}+v_{2}\right)+g v_{1} G_{1} v_{2} G_{2}\left(v_{1}+v_{2}\right) \\
& g V_{2}=g V_{1}+g v_{2}+g V_{1} g v_{2}+g v_{2} g V_{2}+g V_{1} g v_{2} g V_{2} \\
& V_{2}=V_{1}+v_{2}+V_{1} g v_{2}+v_{2} g V_{2}+V_{1} g v_{2} g V_{2} \\
& V_{2}=V_{1}+\left(1+V_{1} g\right)\left(v_{2}+v_{2} g V_{2}\right) .
\end{aligned}
$$

First (9) is multiplied by $\left(v_{1}+v_{2}\right)$. From the first to the second line in the term $G_{1} v_{2}$ as well as the last term the development $G_{1}=g+g v_{1} G_{1}$ has been used. From the second to the third line on the left the conversion is $G_{2}\left(v_{1}+v_{2}\right)=g V_{2}$ according to at once full inclusion of all interactions in the Green's functions. On the right we needed $G_{1} v_{1}=g V_{1}$ and $v_{1} G_{1}=V_{1} g$ as well as again $G_{2}\left(v_{1}+v_{2}\right)=g V_{2}$. A factor $g$ on the left of all terms is skipped in the end. Obviously the right-hand side of (16) is not $v_{2}+v_{2} g V_{2}$ and in (13) replacing $V$ by $V_{2}$ and $v$ by $V_{1}+v_{2}$ or even by $V_{1}+v_{2}+V_{1} g v_{2}$ does not yield the correct result either, as discussed in the appendix. The additional line after (16), however, gives a possibility to represent the Dyson equation for $V_{2}$ wherein $V_{1}$ on the one hand and $v_{2}$ and $V_{2}$ on the other hand appear in well separated parts.

\section{Solving the Dyson equations}

Solving Dyson equations, of course, is complicated by them being implicit integral equations. In actual calculations, however, integrals get replaced by finite sums, which then makes $V(\xi)$ a vector and $V\left(\xi, \xi^{\prime}\right), g\left(\xi, \xi^{\prime}\right)$ and $G\left(\xi, \xi^{\prime}\right)$ matrices [9], or the convolution-like integrals are turned into simple products of functions by using Fourier transforms of $g, v, G$ and $V[7,12]$. In both cases then the Dyson equations are solved by mere algebra as subliminally already suggested by their short forms. We list the results as needed to establish either the normal or the transfer Green's functions in a two-step procedure.

$$
\begin{aligned}
& G_{1}=\left(\mathbf{1}-g v_{1}\right)^{-1} g \\
& G_{2}=\left(\mathbf{1}-G_{1} v_{2}\right)^{-1} G_{1} \\
& V_{1}=\left(\mathbf{1}-v_{1} g\right)^{-1} v_{1} \\
& V_{2}=\left(\mathbf{1}-v_{2} g-V_{1} g v_{2} g\right)^{-1}\left(V_{1}+v_{2}+V_{1} g v_{2}\right) .
\end{aligned}
$$

Some technical details to remark: 1 means the unit matrix or simply the number one, whichever is appropriate. If $v_{1}$ and $v_{2}$ become a list of numbers associated with a set of $\xi$ because the $\xi$ space is discretized into finite elements, a vector space suffices to store these functions, but in equations (17)-(20) they have to be implied as the corresponding diagonal matrices. In the case that the original problem already involves different sites in space and interactions $v$ then invoke coupling between these, the transfer functions are conveniently set up as the respective non-diagonal and full matrices (see section 7). The Dyson equations and their solutions have been given in their most general form here. 


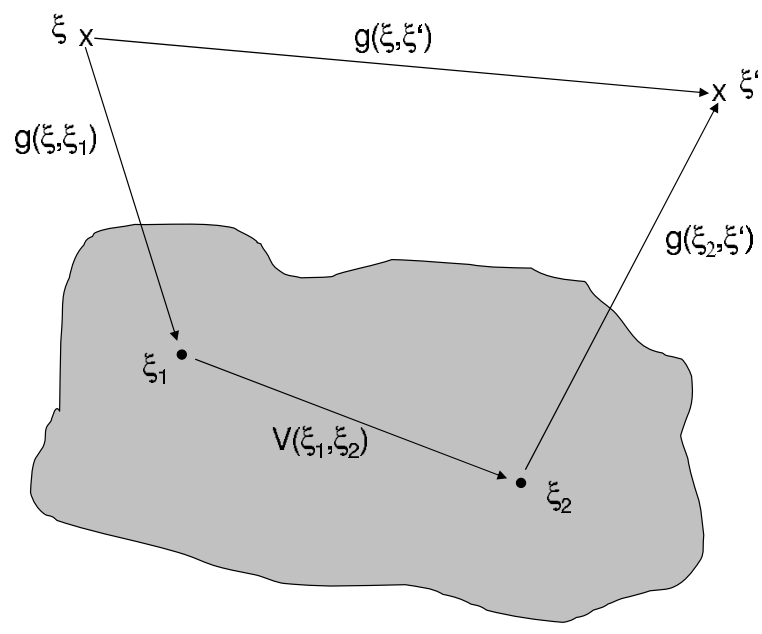

Figure 1. Illustration of equation (21). The effect an elementary excitation at $\xi$ has at $\xi^{\prime}$ consisting of the direct path and the path via the perturbations to the background system. ( $\xi$ or $\xi^{\prime}$ could as well be in the gray area.)

\section{Link to the self-energy}

Especially if the problem consists in determining electron states or matrix elements between them with a scattering potential, $v$ from (12), commonly written $\Sigma$, is called the self-energy. If $D_{\xi}$ in the original differential equation is an eigenenergy minus the Hamiltonian $E-H$, the equation with perturbation, that is (4) with setting $v_{1}=\Sigma$, is often solved in the way of giving the Green's function as an inverse operator $G=(E-H-\Sigma)^{-1}$ [6]. For the background and full system's Green's functions $g$ and $G$ there are the corresponding differential equations (2) and (4) without and with perturbation. There is the Dyson equation (13) for the transfer function $V$ in complete analogy to (12) for G. Nolting discusses it as Dyson equation for the density correlation [4] (chapter 5.6). However, there is no meaningful representation of the differential equation (4) with $V$ and $g$ instead of $v$ and $G$. $V$ can be understood as a renormalized self-energy when writing $G$ as

$$
G=g+g V g
$$

that is comprising the infinite series $G=g+g v g+g v g v g+g v g v g v g+\cdots$ in a form like the first two terms only. Economou has such a notion of self-energy [5] (chapter 10.4) and the quantity called $\Sigma$ here, equal to $v_{1}$ or $v_{1}+v_{2}$ in our problems, should more precisely be called irreducible or proper self-energy.

In contrast to $g$ and $G, v$ is a function of only one $\xi$ variable whereas $V$ is of $\xi$ and $\xi^{\prime} . \xi$ actually being space variables, $V$ can be viewed as non-local potential. The electrodynamics application of the Green's function formalism [3], where the wave equation for the electric field is the differential equation to start with and $v$ is the deviation of a position-dependent $\varepsilon(\vec{r})$ from the background dielectric constant, can serve as a direct illustration. $V$ is a measure of the excitation an effect at $\xi_{1}$ in the whole set of scatterers has at $\xi_{2}$, such that (21), of course integrating over $\xi_{1}$ and $\xi_{2}$, gives the complete influence of an excitation at any $\xi$ anywhere else at $\xi^{\prime}$ (figure 1). By the way, an example of a problem with two different kinds of perturbations which can be treated by folding Green's functions into one another as described by (18) is a setup of scatterers with dielectric and magnetic susceptibilities [9]. In 
the literature about Green's functions in electrodynamics the normal function $G$ is used, not $V$ as defined here, and changing the formalism would not bring any advantage in calculations of field distributions and alike problems. The concept of including perturbations successively has already long been applied in exactly this field, though, even for many more than two. The recipe to construct the Green's function for a configuration of scatterers is repeated use of (9) as $G_{n}=G_{n-1}+G_{n-1} v_{n}\left(1-G_{n-1} v_{n}\right)^{-1} G_{n-1}$ adding scatterer $v_{n}$ in step number $n$ [3, 9 (electronic supplement), 11].

In the quantum transport application we shall discuss below, $V$ will be denoted $T$ and, although in a Fourier representation be resolved into energy-dependent coefficients and phase factors, essentially remains a function of two time arguments. An interpretation as an energy or potential has no meaning in the non-equilibrium situation with an applied voltage driving a current through one or several junctions. Not to be confused with a transmission (probability) function, eventually but for some trivial phase factors or multiplying with densities of states, our $T$ is better identified to the (amplitude) scattering matrix $s$ [6] (chapter 3).

\section{Application}

\subsection{Introduction}

As the example where the second-order Dyson equation for the transfer function is effectively used-and which had led us to its deduction-we discuss coherent charge transport through a superconducting double junction [10]. Properties and results for the single junction [7] are recapitulated as a preparation. The constriction shall be described by a so-called transport channel with transmission amplitude $t(0<t \leqslant 1)$. Most realistic quantum point contacts accommodate several independent channels [13, 14], but for simplicity we restrict the investigation here to one, and later on to one channel per junction in the double-junction system. The interference of multiple reflections across the junction has to be taken into account. In the normal-conducting state the transmission probability $\theta$ is given by the square of a renormalized transmission amplitude, $\theta=\frac{4 t^{2}}{\left(1+t^{2}\right)^{2}}$, and the current is proportional to the applied voltage: $I=\frac{4 t^{2}}{\left(1+t^{2}\right)^{2}} \frac{e^{2}}{h} V$. With a constant density of states assumed in the model, this result simply reflects the overlap on the energy axis of occupied electron states below the Fermi energy on one side and empty states above it on the other. $\frac{e^{2}}{h}$ is the so-called quantum conductance [15]. Zero temperature is assumed.

\subsection{Single junction and Andreev reflection}

Whereas in the normal state even multiple reflections do not mix energy levels, Andreev reflection complicates the picture for the superconducting state. Our calculation will include multiple Andreev reflection (MAR), but no Cooper-pair tunneling [16]. Conductance from electrons as well as from holes has to be considered. In a potential diagram Andreev reflection is viewed as the conversion of an electron into a hole or vice versa and mirroring the energy level at the Fermi level at the side the original particle is going to. Holes should be referred to a reversed energy axis and they have occupied states above and empty ones below the gap in both superconductors L and R. During a MAR the level of the charge carrier can come anywhere outside or inside the gap on either side. The electron in the process from $x_{2}$ in figure 2 is depicted being in the middle of a MAR process. The process from $x_{1}$ can be a completed Andreev reflection as drawn. The electron originates from the filled reservoir below the gap on the left side and the hole finds an empty state in that same reservoir $\left(x_{1}\right.$ in figure $2(b)$ is a negative quantity). With the reversed energy axis for holes, the mirroring 


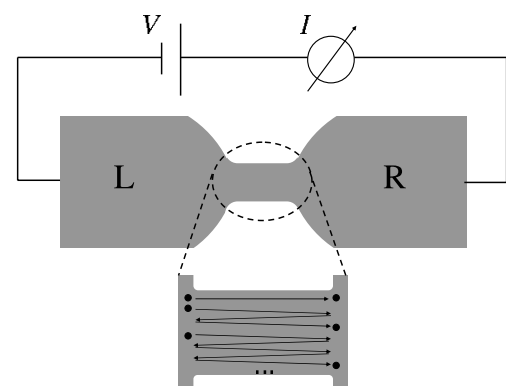

(a)

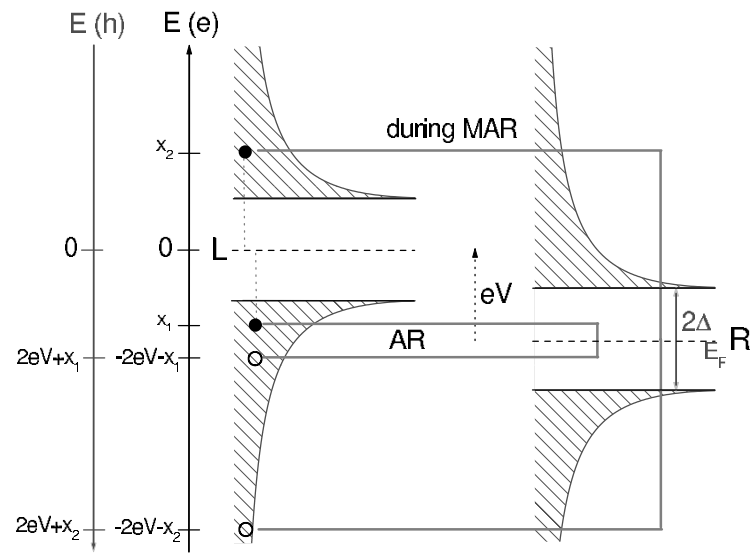

(b)

Figure 2. (a) A narrow junction (point contact) between a left and right lead embedded in an electrical circuit and charge transfers across the constriction in multiple reflections of increasing order. $(b)$ BCS density of states for superconducting reservoirs left and right with voltage drop $V$ between them. Energy axis for electrons and holes, a single Andreev reflection and one out of a MAR process. AR can be viewed as electron-hole conversion and mirroring at the Fermi energy level.

picture tells us that by MAR each energy level gets connected to a discrete ladder of those lying multiples of $2 e V$ away. (As the simplest example the AR in figure 2(b) goes from $x_{1}$ to $2 e V+x_{1}$.) Andreev reflections can be preceded, followed or interluded by single or multiple hoppings across the junction of an electron (or hole) remaining an electron (or hole) and staying at constant energy. The model automatically includes these mixed processes.

In the transport problem we have to distinguish advanced and retarded Green's functions and all the Dyson equations from sections 2-6 are valid for either sort. Similar to the notation in [9] we used $v$ and $V$ for the general formalism, but shall now call these quantities $\sigma$ and $T$, respectively. Primarily the Green's and transfer functions are functions of time ( $\xi$ is now called $\tau$ ), but the calculation works with Fourier representations. For example:

$$
g^{r / a}\left(\tau, \tau^{\prime}\right)=\frac{1}{2 \pi} \int_{-\infty}^{\infty} \mathrm{d} \omega g^{r / a}(\omega) \mathrm{e}^{-\mathrm{i} \omega \tau} \mathrm{e}^{\mathrm{i} \omega \tau^{\prime}}
$$

The background Green's function describing a bulk superconductor such as the left or the right reservoir without the connection between them is (skipping a constant for scaling to the density of states per frequency interval $[7,11])$

$$
\left(\begin{array}{ll}
g_{e e} & g_{e h} \\
g_{h e} & g_{h h}
\end{array}\right)_{L L / R R}^{r / a}(\omega)=\frac{1}{\sqrt{(\Delta / \hbar)^{2}-(\omega \pm \mathrm{i} \eta)^{2}}}\left(\begin{array}{cc}
-\omega \mp \mathrm{i} \eta & \Delta / \hbar \\
-\Delta / \hbar & \omega \pm \mathrm{i} \eta
\end{array}\right) .
$$

$\eta$ is an auxiliary small imaginary part to $\omega$ to determine the sign of the root. Smoothing the singularities in the BCS density of states at the edges of the gap is not absolutely necessary. ( $\hbar \eta / \Delta=1 \times 10^{-7}$ in the example calculations shown.) Multiple signs in (23) refer to the retarded and advanced function in that order. Being a full matrix in electron-hole space, $g$ includes the conversion for Andreev reflection. The hopping function $\sigma$ only has separated electron and hole components. In contrast to the density of states (see figure $2(b)$ ), although 


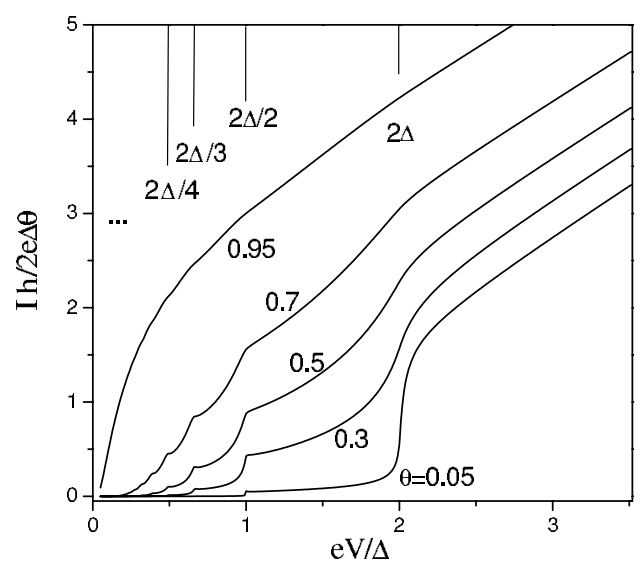

(a)

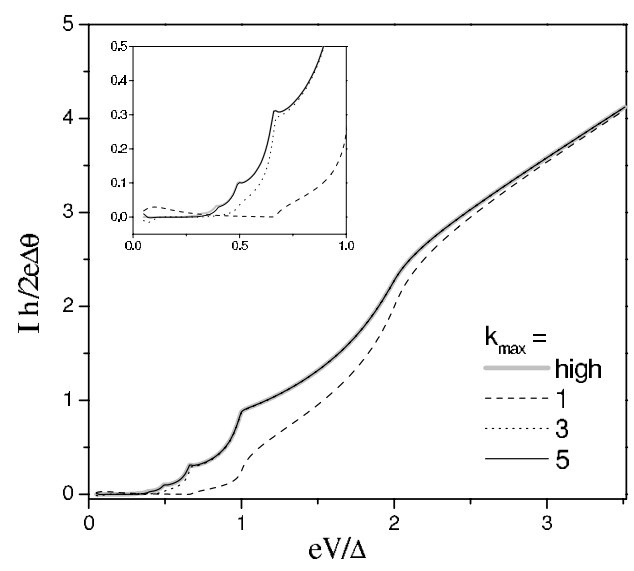

(b)

Figure 3. (a) Normalized calculated current-voltage $(I V)$ curves for the single junction from figure 2 with a single transport channel of transmission $\theta$. Different values of $\theta$. (b) $I V$ for $\theta=0.5$ and calculation repeated for lower limits on the recursion parameter $k$. Inset: magnification of the low-voltage region.

changing from imaginary to real values, $g$ does not vanish inside the gap. For the hopping function we have:

$$
\left(\begin{array}{cc}
\sigma_{e e} & 0 \\
0 & \sigma_{h h}
\end{array}\right)_{L R / R L}=\left(\begin{array}{cc}
t \mathrm{e}^{ \pm \mathrm{i} e V \tau / \hbar} & 0 \\
0 & -t \mathrm{e}^{\mp \mathrm{i} e V \tau / \hbar}
\end{array}\right) .
$$

The phases have to fit to the time development of the superconductor wavefunctions left and right. Directions LR and RL differ. $\sigma$ does not distinguish the advanced and retarded cases, whereas $T$ will. For a left and a right reservoir with connection the original Schrödinger equation already is a matrix equation in the site space consisting of left( $\mathrm{L})$ and $\operatorname{right}(\mathrm{R})$, with $\sigma$ being the coupling part of the Hamiltonian. In this site space we have $g=\left(\begin{array}{cc}g_{L L} & 0 \\ 0 & g_{R R}\end{array}\right)$ and $\sigma=\left(\begin{array}{cc}0 & \sigma_{L R} \\ \sigma_{R L} & 0\end{array}\right)$, and we shall further introduce $T=\left(\begin{array}{cc}T_{L L} & T_{L R} \\ T_{R L} & T_{R R}\end{array}\right)$. The also full matrix $G=\left(\begin{array}{ll}G_{L L} & G_{L R} \\ G_{R L} & G_{R R}\end{array}\right)$ is not needed here just for stating the resulting current formula. If written without LR-indices, there are $2 \times 2$ matrices in site space behind the Green's and transfer functions. There further is a $2 \times 2$ matrix in $e h$-space behind each site space element of $g, \sigma, T$ or $G$, although we rarely explicitly write that. Once having obtained $T^{r / a}(\omega)$ the current is evaluated as

$$
\begin{aligned}
I=e \int_{-\infty}^{\infty} \mathrm{d} \omega & 2 \operatorname{Re} \sum_{k=-\infty}^{\infty} \operatorname{Tr}\left[T_{R L}^{r, k}(\omega) g_{L L}^{+-}\left(\omega+k \frac{e V}{\hbar}\right) T_{L R}^{a, k}(\omega) g_{R R}^{a}(\omega)\right. \\
& \left.+g_{L L}^{r}\left(\omega+k \frac{e V}{\hbar}\right) T_{L R}^{r, k}(\omega) g_{R R}^{+-}(\omega) T_{R L}^{a, k}(\omega)\right]
\end{aligned}
$$

where $g^{+-}(\omega)=g^{a}(\omega)-g^{r}(\omega)$ for $\omega<0$ and $g^{+-}(\omega)=g^{r}(\omega)-g^{a}(\omega)$ for $\omega>0$. Some calculated current-voltage curves for the single junction are shown in figure 3(a). They are in excellent agreement with experimental results [14] for single-atom contacts. For medium $\theta$ they exhibit steps at submultiples $\frac{2 \Delta}{n e}$ of the gap ( $n$ integer) for the applied voltage that stem from Andreev reflection and higher-order MAR processes. In figure $3(b)$ the $I V$ curve for $\theta=0.5$ is shown together with the results for very low limits in the calculation on the $k$ index, the meaning of which will become clear in the next subsection. $k=5$ is acceptable with this 
$\theta, k=3$ merely cuts some finer steps, but $k=1$ is sufficient only for high voltages beyond $2 \Delta / e$. The algorithm is made such that even this lowest-order limit does not completely miss Andreev reflections, however, clearly gives much too low sub-gap current and even spurious results for very small $V$. We shall not derive the current formula (25) here, but need to summarize how $T$ is calculated by a recursion [7].

\subsection{Calculating the T-function}

Equation (13) for the specific problem of the single-channel junction reads

$$
\left(\begin{array}{cc}
T_{L L} & T_{L R} \\
T_{R L} & T_{R R}
\end{array}\right)=\left(\begin{array}{cc}
0 & \sigma_{L R} \\
\sigma_{R L} & 0
\end{array}\right)+\left(\begin{array}{cc}
0 & \sigma_{L R} \\
\sigma_{R L} & 0
\end{array}\right)\left(\begin{array}{cc}
g_{L L} & 0 \\
0 & g_{R R}
\end{array}\right)\left(\begin{array}{cc}
T_{L L} & T_{L R} \\
T_{R L} & T_{R R}
\end{array}\right) .
$$

As the procedure is alike for all components, and in calculating those appearing in (25), that is LR and RL as both retarded and advanced, $T_{L R}^{r}$ is looked upon in an exemplary way (skip superscript $r$ from now on). Writing (26) componentwise and inserting these into one another, a Dyson equation for $T_{L R}$ alone is extracted:

$$
\begin{aligned}
& T_{L R}=\sigma_{L R}+\sigma_{L R} g_{R R} \sigma_{R L} g_{L L} T_{L R} \\
& \text { or } \\
& \begin{aligned}
T_{L R}\left(\tau, \tau^{\prime}\right)= & \delta\left(\tau-\tau^{\prime}\right) \sigma_{L R}(\tau) \\
& \quad+\int_{-\infty}^{\infty} \mathrm{d} \tau_{1} \int_{\infty}^{\infty} \mathrm{d} \tau_{2} \sigma_{L R}(\tau) g_{R R}\left(\tau, \tau_{1}\right) \sigma_{R L}\left(\tau_{1}\right) g_{L L}\left(\tau_{1}, \tau_{2}\right) T_{L R}\left(\tau_{2}, \tau^{\prime}\right) .
\end{aligned}
\end{aligned}
$$

Based on the connection of energy levels to those and only those multiples of $e V$ or of $2 e V$ away, the Fourier representation of $T$ can be set up as:

$$
T\left(\tau, \tau^{\prime}\right)=\frac{1}{2 \pi} \sum_{k=-\infty}^{\infty} \int_{-\infty}^{\infty} \mathrm{d} \omega T^{k}(\omega) \mathrm{e}^{-\mathrm{i} \omega \tau} \mathrm{e}^{-\mathrm{i} k e V \tau / \hbar} \mathrm{e}^{\mathrm{i} \omega \tau^{\prime}}
$$

( $k$ is a superscript, not a power.) In $T_{L R}$ exclusively odd $k$ appear. One $e V$ is allowed for changing the reference level from the left Fermi energy to the right one. Some care must be taken which one the argument $\omega$ refers to [11], but here this issue shall be restricted to the remark that making the argument the same for all $T$-functions in (25) is of advantage. Exploiting complex conjugate relations can save some effort in providing them [12].

Now using (24), (22) and (28) in (27) produces

$$
\begin{aligned}
& \left(\begin{array}{cc}
T_{e e} & T_{e h} \\
T_{h e} & T_{h h}
\end{array}\right)^{k}(\omega)=\delta_{k, 1} \underbrace{\left(\begin{array}{ll}
t & 0 \\
0 & 0
\end{array}\right)}_{t_{1}}+\delta_{k,-1} \underbrace{\left(\begin{array}{cc}
0 & 0 \\
0 & -t
\end{array}\right)}_{t_{-1}} \\
& \underbrace{+t^{2}\left(\begin{array}{cc}
g_{e e}\left(\omega+(k-1) \frac{e V}{\hbar}\right) & 0 \\
0 & g_{h h}\left(\omega+(k+1) \frac{e V}{\hbar}\right)
\end{array}\right)\left(\begin{array}{ll}
g_{e e} & g_{e h} \\
g_{h e} & g_{h h}
\end{array}\right)\left(\omega+k \frac{e V}{\hbar}\right)}_{\epsilon_{k}}\left(\begin{array}{ll}
T_{e e} & T_{e h} \\
T_{h e} & T_{h h}
\end{array}\right)^{k}(\omega) \\
& \underbrace{-t^{2}\left(\begin{array}{cc}
0 & g_{e h} \\
0 & 0
\end{array}\right)\left(\omega+(k-1) \frac{e V}{\hbar}\right)\left(\begin{array}{cc}
0 & 0 \\
g_{h e} & g_{h h}
\end{array}\right)\left(\omega+(k-2) \frac{e V}{\hbar}\right)}_{V_{k}^{-}}\left(\begin{array}{ll}
T_{e e} & T_{e h} \\
T_{h e} & T_{h h}
\end{array}\right) \quad(\omega) \\
& \underbrace{-t^{2}\left(\begin{array}{cc}
0 & 0 \\
g_{h e} & 0
\end{array}\right)\left(\omega+(k+1) \frac{e V}{\hbar}\right)\left(\begin{array}{cc}
g_{e e} & g_{e h} \\
0 & 0
\end{array}\right)\left(\omega+(k+2) \frac{e V}{\hbar}\right)}_{V_{k}^{+}}\left(\begin{array}{cc}
T_{e e} & T_{e h} \\
T_{h e} & T_{h h}
\end{array}\right)^{k+2}(\omega)
\end{aligned}
$$


(29) is a relation for $T$ as functions of frequency and has to be solved for each $\omega$. With $z_{k}^{+}$and $z_{k}^{-}$defined by

$$
T^{k+2}=z_{k}^{+} T^{k} \quad \text { and } \quad T^{k-2}=z_{k}^{-} T^{k}
$$

these are evaluated as

$z_{k-2}^{+}=\left(\mathbf{1}-\epsilon_{k}-V_{k}^{+} z_{k}^{+}\right)^{-1} V_{k}^{-} \quad$ and $\quad z_{k+2}^{-}=\left(\mathbf{1}-\epsilon_{k}-V_{k}^{-} z_{k}^{-}\right)^{-1} V_{k}^{+}$

cutting the range of $k$ such that for a maximum positive $k$ the term $V_{k}^{+} z_{k}^{+}$is set to zero as well as $V_{k}^{-} z_{k}^{-}$for a minimum negative $k . z_{k}^{+}$is thus provided going down to $k=1$ for positive $k$ ( $k$ is odd) and $z_{k}^{-}$is obtained going up to $k=-1$ for negative $k$. $T^{k}$ is then initiated by solving

$$
\begin{aligned}
& T^{1}=t_{1}+\epsilon_{1} T^{1}+V_{1}^{+} z_{1}^{+} T^{1}+V_{1}^{-} T^{-1} \\
& T^{-1}=t_{-1}+\epsilon_{-1} T^{-1}+V_{-1}^{+} T^{1}+V_{-1}^{-} z_{-1}^{-} T^{-1} .
\end{aligned}
$$

From these all other $T^{k}$ up to the limits of $k$ are easily evaluated using (30). It is interesting to note that the necessity to cut the $k$-range to finite values does not limit the order of the multiple (Andreev) reflection processes included. It restricts the energy difference a process can go away from the Fermi energies or the gap edges left and right. As larger energy differences require electron-hole conversion and the off-diagonal components $g_{e h}$ and $g_{h e}$ tend to zero for $\omega \rightarrow \pm \infty$, the error can be made negligible. Smaller voltages $V$ require higher $k$-limits. Through the inward-recursion for $z_{k}^{ \pm}$preceding the outward one to calculate the $T^{k}$, even $T^{k}$ for small $k$ depend on the $k$-limit. This seems a subtle effect in some narrow peaks, however, the evaluation of the current includes calculating the area under such peaks. Figure 4 as an example shows a single component of a transfer function as well as the integrand for the current function in (25), which but for two more $g$-factors is kind of a squared absolute amplitude of the transfer function.

\subsection{Double junction and difficulties getting $T$}

As besides the general derivation in section 4 is the main purpose of this paper, we shall now extend the Green's functions formalism for the transport problem to the double junction (figure 5). We assume a sufficiently small island between the two junctions, such that coherence can be maintained in transport across it, but large enough and bulk-like, such that a few excess charges do not alter the BCS density of states or the occupation given by the Fermi function. To actually calculate current-voltage characteristics, with the interest in whether Coulomb blockade suppresses MAR, the island's Fermi energy level needs to be changed with island charging as is done in $[10,12]$. However, in order to discuss unadulteratedly the recipe to establish the transfer Green's function, the island shall here be assumed to stay at fixed potential. Transport does not merely consist of coherent processes beginning in the left lead and ending in the right or vice versa. Coherent multiple (Andreev) reflection processes extend over both junctions. Nevertheless, they can start or end on the island, which acts as a reservoir like the leads. Sequential transport with relaxation on the island is equally present. Formula like (25) —although having more terms [10, 17] — for the double junction give rates for the island to change its excess charge. A stationary state is then found from a system of classical rate equations. The transfer Green's function is needed anyway, and its determination is the problem to focus on here. With three sites the complete Dyson equation à la equation (15) 

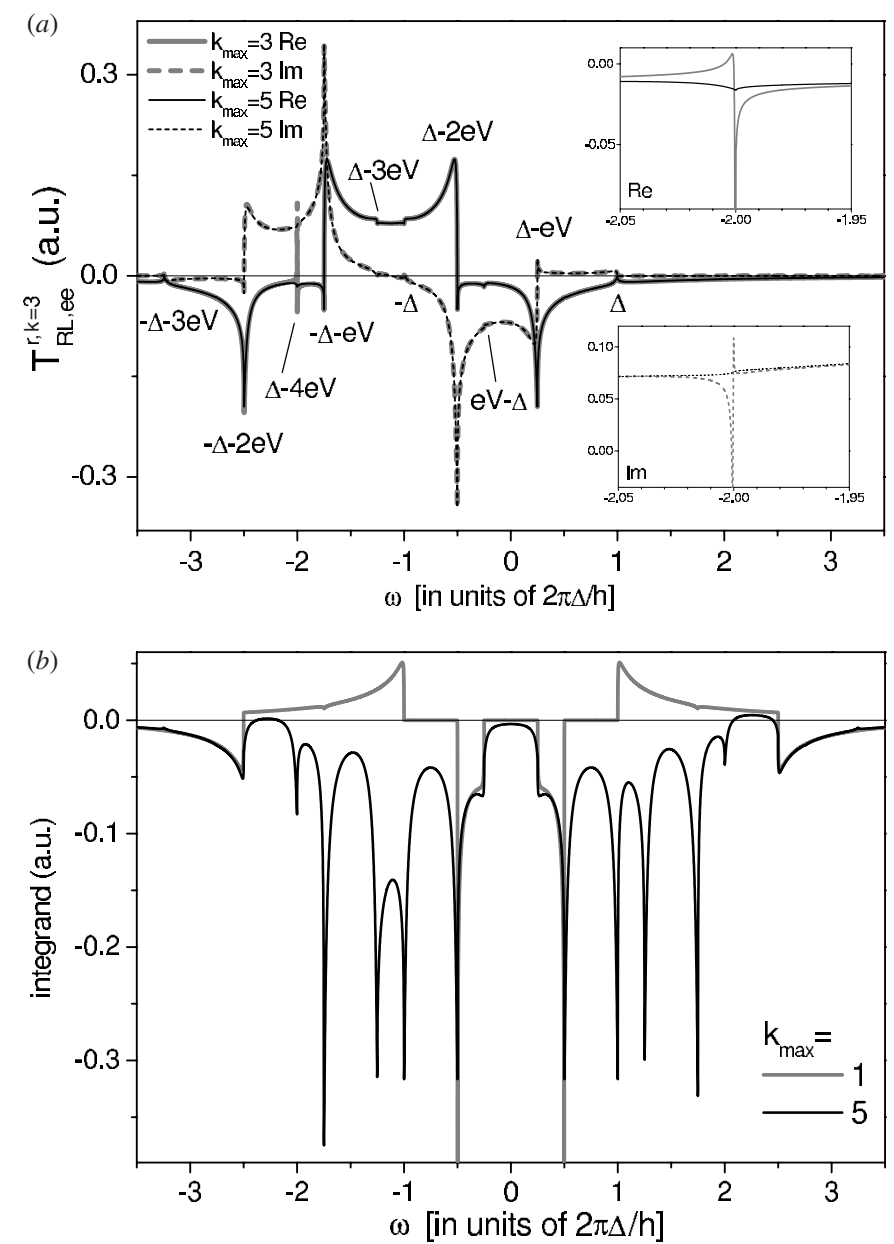

Figure 4. (a) Single-junction transfer function $T_{R L, e e}^{r, k=3}(\omega)$ for $\theta=0.5$ at $V=0.75 \Delta / e$, $\operatorname{Re}$ (solid lines), Im (dashed lines); thick gray lines are results with highest $k=3$, thin black lines with highest $k=5$. Insets: peak at $\omega=-2 \Delta / \hbar$ that is drastically smoothed in more accurate calculation. Peak positions are labeled as multiples of $e V$ plus/minus $\Delta$ (compare energy axis in figure $2(b)$ ). $(b)$ The complete integrand from equation (25), that is both terms for all included $k$ from all possible combinations of $e h$-components, as a function of $\omega$ again for $\theta=0.5$ at $V=0.75 \Delta / e$. For the too-low $k$-limit 1 the integrand in some ranges even has the wrong sign, whereas for the acceptable limit 5, the peak structure reflects that all essential MAR are included. The fact that the complete integrand in contrast to a single $T$-component is symmetric around $\omega=0$ is due to the addition of electron and hole contributions.

reads as

$$
\begin{gathered}
\left(\begin{array}{ccc}
T_{L L} & T_{L I} & T_{L R} \\
T_{I L} & T_{I I} & T_{I R} \\
T_{R L} & T_{R I} & T_{R R}
\end{array}\right)=\left(\begin{array}{ccc}
0 & \sigma_{L I} & 0 \\
\sigma_{I L} & 0 & \sigma_{I R} \\
0 & \sigma_{R I} & 0
\end{array}\right) \\
+\left(\begin{array}{ccc}
0 & \sigma_{L I} & 0 \\
\sigma_{I L} & 0 & \sigma_{I R} \\
0 & \sigma_{R I} & 0
\end{array}\right)\left(\begin{array}{ccc}
g_{L L} & 0 & 0 \\
0 & g_{I I} & 0 \\
0 & 0 & g_{R R}
\end{array}\right)\left(\begin{array}{ccc}
T_{L L} & T_{L I} & T_{L R} \\
T_{I L} & T_{I I} & T_{I R} \\
T_{R L} & T_{R I} & T_{R R}
\end{array}\right)
\end{gathered}
$$




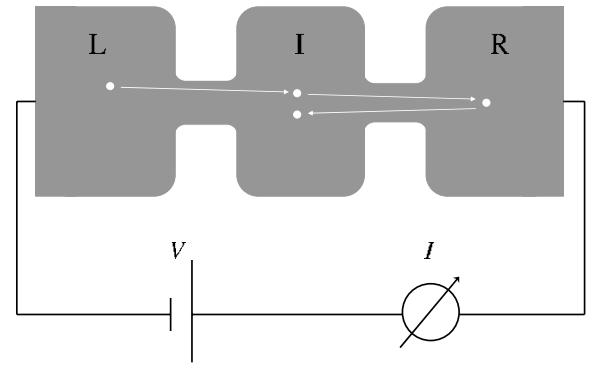

$(a)$
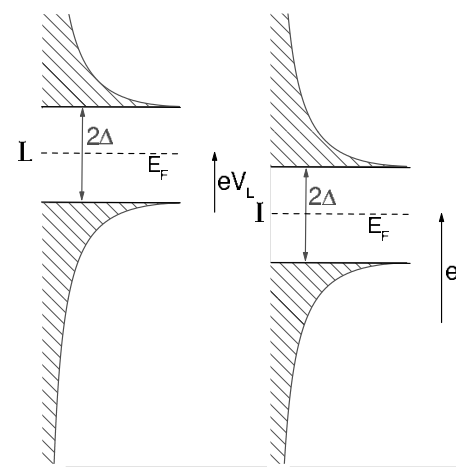

(b)

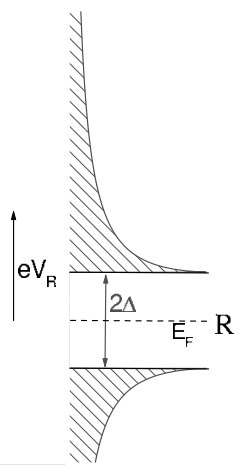

Figure 5. (a) Structure with two junctions and three reservoirs left (L), island (I) and right (R). Coherent multiple reflections and MAR may extend over both junctions. (b) Superconductor densities of states (DOS) for all three reservoirs with voltage drop $V_{L}$ and $V_{R}$, respectively, over the junctions. $V_{L}+V_{R}=V$ and $V_{L}: V_{R}$ is determined by assigning a capacitance to each junction. Shifts of the complete DOS of the island due to charges on it changing its electrical potential are ignored here.

According to the setup there is no direct coupling between L and R. As Andreev reflections can alter the energy level by twice the voltage equivalent dropping over the left junction or twice that over the right junction, for all $T$-components the ansatz as Fourier representation is

$T\left(\tau, \tau^{\prime}\right)=\frac{1}{2 \pi} \sum_{k=-\infty}^{\infty} \sum_{p=-\infty}^{\infty} \int_{-\infty}^{\infty} \mathrm{d} \omega T^{k, p}(\omega) \mathrm{e}^{-\mathrm{i} \omega \tau} \mathrm{e}^{-\mathrm{i} k e V_{L} \tau / \hbar} \mathrm{e}^{-\mathrm{i} p e V_{R} \tau / \hbar} \mathrm{e}^{\mathrm{i} \omega \tau^{\prime}}$.

Analogously to (27), writing (33) as 9 component equations and putting them into one another, implicit equations for single $T$-components can be derived, for example,

$$
T_{I R}=\sigma_{I R}+\sigma_{I L} g_{L L} \sigma_{L I} g_{I I} T_{I R}+\sigma_{I R} g_{R R} \sigma_{R I} g_{I I} T_{I R}
$$

In the same way that (29) was got from (27), an equation like (35) translates into the form

$$
\begin{gathered}
T^{k, p}=\delta_{p, 1} t_{R, 1}+\delta_{p,-1} t_{R,-1}+\epsilon_{k, p} T^{k, p}+V_{k}^{+}(p) T^{k+2, p}+V_{k}^{-}(p) T^{k-2, p} \\
+V_{p}^{+}(k) T^{k, p+2}+V_{p}^{-}(k) T^{k, p-2}
\end{gathered}
$$

for functions $T^{k, p}$ of each frequency $\omega$. Components $T_{R I}$ and $T_{I R}$ have odd $p$ and even $k$ and therefore seed terms $\delta_{p, 1} t_{R, 1}$ and $\delta_{p,-1} t_{R,-1}$. $T_{L I / I L}$ would have $\delta_{k, 1} t_{L, 1}$ and $\delta_{k,-1} t_{L,-1}$, for $T_{L R / R L}$ we would have to take $\delta_{k, \pm 1} t_{L, \pm 1} \cdot \delta_{p, \pm 1} t_{R, \pm 1}$, for $T_{I I} \delta_{k, s} t_{L, s}$ and $\delta_{p, s} t_{R, s}$ with $s=-2,0,2$ and for $T_{L L}$ and $T_{R R}$ only one of those, respectively. (The form (36) only works for $T_{I R}, T_{I L}$ and $T_{I I}$, however.) As coefficients $\epsilon$ and $V^{ \pm}$are given through $g$, equations (36) for all $(k, p)$ can be taken as a linear system of equations and solved by a matrix inversion. It is not even necessary to separate Dyson equations into those for individual $T$-components in site space. As relations for functions of $\omega$, matrix inversion can directly be applied to (26) or (33), having each $T^{k, p}$ connected to other components $T^{k \pm 1, p}$ or $T^{k, p \pm 1}$ in site space. Because of the many-fold combinations of $e h$-components the matrices to invert become rather big, though sparse, already for quite modest limits on $k$ and $p$. We mentioned this direct approach to illustrate that working with an ansatz like (14) or (15) putting all interactions in at once is not in principle impossible, however, is likely to become cumbersome, especially with respect to the practical aspect of creating efficient computer codes. 
However, even if working with the couplings across the left and the right junctions at the same time, (36) in analogy to the calculation for the single junction suggests attempting to evaluate the $T^{k, p}$ by a recursion, which does not require inversion of large matrices. With definitions for $z^{+}$

$$
T^{k+2, p}=z_{k}^{+}(p) T^{k, p} \quad \text { and } \quad T^{k, p+2}=z_{p}^{+}(k) T^{k, p}
$$

in (36) leading all $T$ back to $T^{k-2, p-2}$ for $p \neq \pm 1$ results in

$$
\begin{aligned}
z_{p-2}^{+}(k) z_{k-2}^{+}(p & -2)=\epsilon_{k, p} z_{p-2}^{+}(k) z_{k-2}^{+}(p-2)+V_{k}^{+}(p) z_{k}^{+}(p) z_{p-2}^{+}(k) z_{k-2}^{+}(p-2) \\
& +V_{k}^{-}(p) z_{p-2}^{+}(k-2)+V_{p}^{+}(k) z_{p}^{+}(k) z_{k-2}^{+}(p) z_{p-2}^{+}(k-2)+V_{p}^{-}(k) z_{k-2}^{+}(p-2) .
\end{aligned}
$$

Intermingling the raising and lowering of the $k$ - and $p$-index during the recursion is in principle conceivable, however, even exploiting the necessity that $z_{p-2}^{+}(k) z_{k-2}^{+}(p-2)=$ $z_{k-2}^{+}(p) z_{p-2}^{+}(k-2)$ does not help to decide whether to rearrange (38) as to give an expression for $z_{k-2}^{+}(p-2)$ or one for $z_{p-2}^{+}(k-2)$ and how to evaluate either yet being ignorant of the other. $z_{k}^{-}(p)$ and $z_{p}^{-}(k)$ can be defined in obvious analogy to (37). Products of $z^{+}$'s may be avoided by expressing all $T$ in (36) by $T^{k, p}$ using $T^{k-2, p}=\left(z_{k-2}^{+}(p)\right)^{-1} T^{k, p}$ and $T^{k, p-2}=\left(z_{p-2}^{+}(k)\right)^{-1} T^{k, p}$. This attempt-the same as trying to use inverse $z$ in (38)equally runs into a dead end having the two unknowns $z_{k-2}^{+}(p)$ and $z_{p-2}^{+}(k)$ in the recursion relation.

\subsection{Two-step procedure for $T$}

Since the two-dimensional recursion in $k$ and $p$ (in analogy to the single-junction case) does not work out, we now develop an algorithm taking into account the couplings across the left and right junctions one after another. First a transfer Green's function now called $T^{1}$ is made for the system with only the connection between $L$ and I present applying exactly the algorithm known from the single junction. However, instead of $T_{L I}$ in analogy to $T_{L R}$ from the single junction, it comes in handy to select the Dyson equation reduced to the component $T_{I I}^{1}$. For the second step adding the connection between I and R, the Dyson equation to use is (16). All quantities are $3 \times 3$ matrices in site space in this case. $V_{2}$ is the full $T$-matrix as in (33). $v_{2}$ only has entries $\sigma_{I R}$ and $\sigma_{R I}$ and zeros everywhere else. $g$, of course, is diagonal with $g_{L L}, g_{I I}$ and $g_{R R}$. $V_{1}$ with entries $T_{L L}^{1}, T_{L I}^{1}, T_{I L}^{1}$ and $T_{I I}^{1}$ has to be completed by a third line and column of zeros. Thus with the analog of (26) as the first-step Dyson equation

$$
\left(\begin{array}{cc}
T_{L L}^{1} & T_{L I}^{1} \\
T_{I L}^{1} & T_{I I}^{1}
\end{array}\right)=\left(\begin{array}{cc}
0 & \sigma_{L I} \\
\sigma_{I L} & 0
\end{array}\right)+\left(\begin{array}{cc}
0 & \sigma_{L I} \\
\sigma_{I L} & 0
\end{array}\right)\left(\begin{array}{cc}
g_{L L} & 0 \\
0 & g_{I I}
\end{array}\right)\left(\begin{array}{cc}
T_{L L}^{1} & T_{L I}^{1} \\
T_{I L}^{1} & T_{I I}^{1}
\end{array}\right)
$$

the second-step Dyson equation reads as

$$
\begin{aligned}
\left(\begin{array}{ccc}
T_{L L} & T_{L I} & T_{L R} \\
T_{I L} & T_{I I} & T_{I R} \\
T_{R L} & T_{R I} & T_{R R}
\end{array}\right)=\left(\begin{array}{ccc}
T_{L L}^{1} & T_{L I}^{1} & 0 \\
T_{I L}^{1} & T_{I I}^{1} & 0 \\
0 & 0 & 0
\end{array}\right)+\left(\begin{array}{ccc}
0 & 0 & 0 \\
0 & 0 & \sigma_{I R} \\
0 & \sigma_{R I} & 0
\end{array}\right) \\
+\left(\begin{array}{ccc}
T_{L L}^{1} & T_{L I}^{1} & 0 \\
T_{I L}^{1} & T_{I I}^{1} & 0 \\
0 & 0 & 0
\end{array}\right)\left(\begin{array}{ccc}
g_{L L} & 0 & 0 \\
0 & g_{I I} & 0 \\
0 & 0 & g_{R R}
\end{array}\right)\left(\begin{array}{ccc}
0 & 0 & 0 \\
0 & 0 & \sigma_{I R} \\
0 & \sigma_{R I} & 0
\end{array}\right)
\end{aligned}
$$




$$
\begin{aligned}
& +\left(\begin{array}{ccc}
0 & 0 & 0 \\
0 & 0 & \sigma_{I R} \\
0 & \sigma_{R I} & 0
\end{array}\right)\left(\begin{array}{ccc}
g_{L L} & 0 & 0 \\
0 & g_{I I} & 0 \\
0 & 0 & g_{R R}
\end{array}\right)\left(\begin{array}{ccc}
T_{L L} & T_{L I} & T_{L R} \\
T_{I L} & T_{I I} & T_{I R} \\
T_{R L} & T_{R I} & T_{R R}
\end{array}\right) \\
& +\left(\begin{array}{ccc}
T_{L L}^{1} & T_{L I}^{1} & 0 \\
T_{I L}^{1} & T_{I I}^{1} & 0 \\
0 & 0 & 0
\end{array}\right)\left(\begin{array}{ccc}
g_{L L} & 0 & 0 \\
0 & g_{I I} & 0 \\
0 & 0 & g_{R R}
\end{array}\right) \\
& \times\left(\begin{array}{ccc}
0 & 0 & 0 \\
0 & 0 & \sigma_{I R} \\
0 & \sigma_{R I} & 0
\end{array}\right)\left(\begin{array}{ccc}
g_{L L} & 0 & 0 \\
0 & g_{I I} & 0 \\
0 & 0 & g_{R R}
\end{array}\right)\left(\begin{array}{lll}
T_{L L} & T_{L I} & T_{L R} \\
T_{I L} & T_{I I} & T_{I R} \\
T_{R L} & T_{R I} & T_{R R}
\end{array}\right) .
\end{aligned}
$$

Again, from the 9 component equations (40) we insert that having $T_{I I}$ on the left into the expression for $T_{R I}$ to get a Dyson equation for one component, $T_{R I}$ chosen as example here, exclusively:

$$
\begin{aligned}
T_{R I} & =\sigma_{R I}+\sigma_{R I} g_{I I} T_{I I}^{1}+\sigma_{R I} g_{I I} \sigma_{I R} g_{R R} T_{R I}+\sigma_{R I} g_{I I} T_{I I}^{1} g_{I I} \sigma_{I R} g_{R R} T_{R I} \\
& =\sigma_{R I}\left(1+g_{I I} T_{I I}^{1}\right)+\sigma_{R I}\left(1+g_{I I} T_{I I}^{1}\right) g_{I I} \sigma_{I R} g_{R R} T_{R I}
\end{aligned}
$$

Equations for other components of the final transfer function may have a little different structure, especially will there be terms beginning with $T^{1}$ in those for $T$ with first index L or I. In (40) or (41) $T^{1}$ is a known function like $g$ and $\sigma$. Like (29) follows from (27), (41) is transformed into a recursion relation with respect to the $p$-index by inserting representations (34) for $T_{R I}$, (28) for $T_{I I}^{1}$, (22) for $g$ and (24) with $t_{R}$ and $V_{R}$ for $\sigma_{R I / I R}$ :

$$
\begin{aligned}
T^{p}(k)=\delta_{p, \pm 1} t_{ \pm 1}(k) & +\sum_{k^{\prime}} \epsilon_{p}\left(k, k^{\prime}\right) T^{p}\left(k^{\prime}\right) \\
& +\sum_{k^{\prime}} V_{p}^{+}\left(k, k^{\prime}\right) T^{p+2}\left(k^{\prime}\right)+\sum_{k^{\prime}} V_{p}^{-}\left(k, k^{\prime}\right) T^{p-2}\left(k^{\prime}\right)
\end{aligned}
$$

$k$ and $k^{\prime}$ are even. The seed $t_{ \pm 1}(k)$ corresponds to $\sigma_{R I}+\sigma_{R I} g_{I I} T_{I I}^{1}$ here. As the prefactors $\epsilon$ and $V^{ \pm}$can already contribute multiples of the potential drop over the left junction in energy change, the recursion relation directly interlinks $T^{k, p( \pm 2)}$ with all $k$-indices. The $z_{p}^{ \pm}$as well as factors in their calculation are full matrices now with the dimension of the $k$-range (each entry further representing a quadruple of $e h$-space components). Matrix inversion cannot be avoided, however, whereas in the approach for a direct solution of (33) mentioned in the last section we would have had to deal with matrices of size the $k$-times the $p$-range, the size only the $k$-range is much more modest here. We applied a special inversion procedure known from Green's tensors in electrodynamics [9, 11]. In translating the Dyson equation (41) into Fourier space, it is clear that terms $\sigma_{R I}$ and $\sigma_{R I} g_{I I} \sigma_{I R} g_{R R} T_{R I}$ follow the scheme from (29), especially concerning which components of $g$ of which arguments to put (replace $k$ by $p, t$ by $t_{R}$ and $V$ by $V_{R}$ ). These terms only contribute to $t_{ \pm 1}, \epsilon_{p}$ and $V_{p}^{ \pm}$of $k=k^{\prime}$. $\sigma_{R I} g_{I I} T_{I I}^{1}$ gives further contributions $\pm \delta_{p, \pm 1} t_{R} g_{I I}\left(\omega+k \frac{e V_{h}}{\hbar}\right) T_{I I}^{1, k}(\omega)$ to $t_{ \pm 1}(k)$. There are all four components in $e h$-space, namely $g_{e e} T_{e e}^{1}+g_{e h} T_{h e}^{1}$ and $g_{e e} T_{e h}^{1}+g_{e h} T_{h h}^{1}$ (with plus sign) as well as $g_{h e} T_{e e}^{1}+g_{h h} T_{h e}^{1}$ and $g_{h e} T_{e h}^{1}+g_{h h} T_{h h}^{1}$ (with minus sign). From the prefactor of $T_{R I}$ in the last term, that is $\sigma_{R I} g_{I I} T_{I I}^{1} g_{I I} \sigma_{I R} g_{R R}$, there are contributions to $\epsilon_{p}\left(k, k^{\prime}\right)$ if $\sigma_{R I}$ and $\sigma_{I R}$ mean either both an electron or both a hole hopping. $\sigma_{R I}$ being an electron and $\sigma_{I R}$ a hole, the contribution goes into $V_{p}^{-}$, and for $\sigma_{R I}$ a hole and $\sigma_{I R}$ an electron, it is put into $V_{p}^{+}$. Each of these cases still consists of the sum of four possible combinations of $e h$-indices, as for 
example with two electron- $\sigma$ we have for the $e e$-component

$$
\begin{aligned}
\sigma_{e e}^{R I} g_{e e}^{I I} T_{e e}^{1, I I} g_{e e}^{I I} \sigma_{e e}^{I R} g_{e e}^{R R}+\sigma_{e e}^{R I} g_{e e}^{I I} T_{e h}^{1, I I} g_{h e}^{I I} \sigma_{e e}^{I R} g_{e e}^{R R} \\
+\sigma_{e e}^{R I} g_{e h}^{I I} T_{h e}^{1, I I} g_{e e}^{I I} \sigma_{e e}^{I R} g_{e e}^{R R}+\sigma_{e e}^{R I} g_{e h}^{I I} T_{h h}^{1, I I} g_{h e}^{I I} \sigma_{e e}^{I R} g_{e e}^{R R} .
\end{aligned}
$$

In the following we note the components to go into the coefficients as in (29) with frequency arguments. For simplicity of notation we just use $V_{L}$ and $V_{R}$ meaning $e V_{L} / \hbar$ and $e V_{R} / \hbar$. Note that for setting up the equation for $T_{R I}(\omega), T_{I I}^{1}$ is needed of quite a few different frequencies, namely of arguments varying by $\pm 2 V_{R}$ for each $p$ and as many multiples of $V_{L}$ as $k$ involved.

$$
\begin{aligned}
e e: & t_{R}^{2} g_{I I}\left(\omega+k V_{L}+(p-1) V_{R}\right) T_{I I}^{k-k^{\prime}}\left(\omega+k^{\prime} V_{L}+(p-1) V_{R}\right) \cdot \\
& g_{I I}\left(\omega+k^{\prime} V_{L}+(p-1) V_{R}\right) g_{R R}\left(\omega+k^{\prime} V_{L}+p V_{R}\right) \rightarrow \epsilon_{p}\left(k, k^{\prime}\right) \\
e h:-t_{R}^{2} g_{I I}\left(\omega+k V_{L}+(p-1) V_{R}\right) T_{I I}^{k-k^{\prime}}\left(\omega+k^{\prime} V_{L}+(p-1) V_{R}\right) \cdot & \\
& g_{I I}\left(\omega+k^{\prime} V_{L}+(p-1) V_{R}\right) g_{R R}\left(\omega+k^{\prime} V_{L}+(p-2) V_{R}\right) \rightarrow V_{p}^{-}\left(k, k^{\prime}\right) \\
h e: & -t_{R}^{2} g_{I I}\left(\omega+k V_{L}+(p+1) V_{R}\right) T_{I I}^{k-k^{\prime}}\left(\omega+k^{\prime} V_{L}+(p+1) V_{R}\right) \cdot \\
& g_{I I}\left(\omega+k^{\prime} V_{L}+(p+1) V_{R}\right) g_{R R}\left(\omega+k^{\prime} V_{L}+(p+2) V_{R}\right) \rightarrow V_{p}^{+}\left(k, k^{\prime}\right) \\
h h: & t_{R}^{2} g_{I I}\left(\omega+k V_{L}+(p+1) V_{R}\right) T_{I I}^{k-k^{\prime}}\left(\omega+k^{\prime} V_{L}+(p+1) V_{R}\right) . \\
& g_{I I}\left(\omega+k^{\prime} V_{L}+(p+1) V_{R}\right) g_{R R}\left(\omega+k^{\prime} V_{L}+p V_{R}\right) \rightarrow \epsilon_{p}\left(k, k^{\prime}\right) .
\end{aligned}
$$

In figure 6 we calculated one component out of the $T_{R I}$-function with and without interaction corrections due to backreflections from the left lead. Figure $6(a)$ is for a situation where the voltage drops across both junctions each clearly exceed the $2 \Delta$ superconductor gap. $\omega=0$ is the island Fermi level taken for reference, and images of the gaps from the three sites clearly appear distinct from one another. Naturally, that of the left lead is smaller if the left junction is given smaller transmission and disappears if it is ignored by setting $k_{\max }$ to zero. For a $p=1$ part as chosen here, interaction corrections due to hoppings over the left junction do not only alter the peak structure in the transfer function around images of the gap potentials, but also change the constant high and low frequency limit of the real part value. However, apart from a finite interval comprising the peak structures the resulting non-zero integrand for current rates is canceled by corresponding hole contributions in any case, though. More images than the primary ones of the three gap positions are tiny for rather high applied voltage as in figure $6(a)$, but some are still discernable. In figure $6(b)$ where the same $T$-component is shown for applied voltages across the two junctions both less than $2 \Delta / e$, pairs of gap edges from images of the DOS of all three reservoirs become harder to disentangle. In addition to peaks or edges at multiples of the individual voltage drops plus or minus $\Delta$, we find some at positions given by combinations of both voltage drops, which is an evidence for MAR extending over both junctions included in our formula for the transfer function. Sums of products of $T$ for all $k$ and $p$ like in (25) could now be made. However, as mentioned earlier, to realistically model transport through the double junction, frequency arguments of $g$-functions in expressions like (44) would have to be corrected for the island Fermi level changing with the excess charge. With zero charging energy setting up rate equations in order to determine a stationary state does not make sense. The goal of this work was to present the second-step $T$-function equation. 

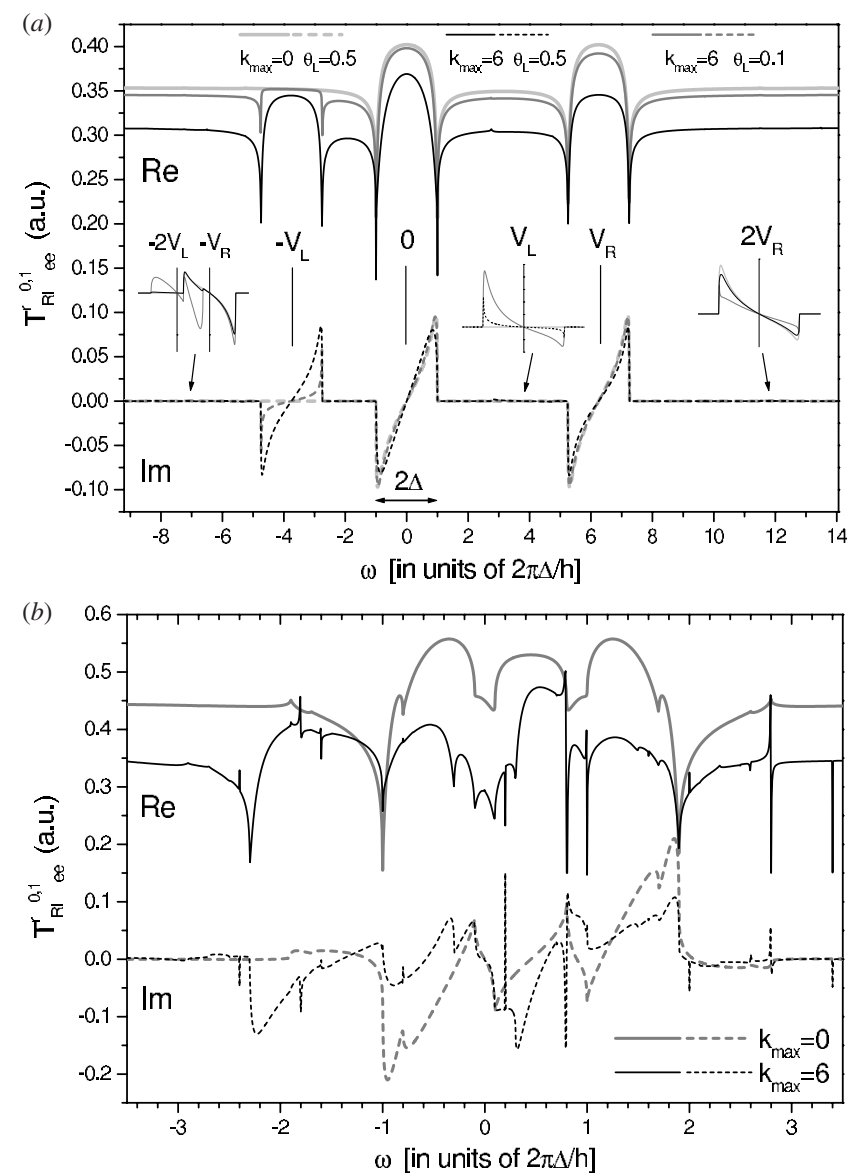

Figure 6. (a) Double-junction transfer function $T_{R I, e e}^{r, k=0, p=1}(\omega)$ for $V_{L}=3.75 \Delta / e$ and $V_{R}=6.25 \Delta / e . \theta_{R}=0.5$ and limit on $p$ in calculation is 5 for all curves. $\theta_{L}$ and limit on $k$ are varied. The real part also shows tiny structures centered around $-2 V_{L},-V_{R}, V_{L}$ and $2 V_{R}$, which are not shown enlarged. (b) Same $T_{R I, e e}^{r, k=0, p=1}(\omega)$ for $V_{L}=1.3 \Delta / e, V_{R}=0.9 \Delta / e, \theta_{L}=\theta_{R}=0.8$ and limit on $p$ is 5 . Limit on $k$ either 6 or 0 . Identification of peaks or edges (not necessarily complete list), numbers are positions on the $\omega$-axis, $V_{L / R}$ is written for $e V_{L / R} / \hbar$ and $\Delta \equiv 1$ : $-2.9=-3 V_{L}+\Delta,-2.7=V_{R}-2 V_{L}-\Delta,-2.5=-V_{R}-2 V_{L}+\Delta,-2.3=-V_{L}-\Delta,-1.9=$ $-V_{R}-\Delta,-1.8=2 V_{R}-2 V_{L}-\Delta,-1.7=-3 V_{R}+\Delta,-1.6=-2 V_{L}+\Delta,-1.0=-\Delta,-0.8=$ $-2 V_{R}+\Delta,-0.3=-V_{L}+\Delta,-0.1=V_{R}-\Delta, 0.1=-V_{R}+\Delta, 0.2=2 V_{R}-2 V_{L}+\Delta, 0.3=$ $V_{L}-\Delta, 0.8=2 V_{R}-\Delta, 1.0=\Delta, 1.5=2 V_{R}-V_{L}+\Delta, 1.7=3 V_{R}-\Delta, 1.9=V_{R}+\Delta, 2.0=$ $4 V_{R}-2 V_{L}+\Delta, 2.3=V_{L}+\Delta, 2.5=V_{R}+2 V_{L}-\Delta, 2.6=4 V_{R}-\Delta, 2.8=2 V_{R}+\Delta$.

\subsection{Additional remarks}

Labeling the left junction as the first and the right junction as the second interaction was an arbitrary choice, regardless of which component (we chose RI as example) of the transfer function is the desired one.

Even for the transport problems like those addressed here, it is not an absolute necessity to use the transfer Green's function. The current formula could be used in its original form

$$
I=e \cdot \operatorname{Tr}\left\{\sigma_{L R} G_{R L}^{+-}-\sigma_{R L} G_{L R}^{+-}\right\} .
$$

(The trace, like in (25), is in $e h$-space.) For the double junction with either $\mathrm{L}$ or $\mathrm{R}$ replaced by I these are probabilities to charge and decharge the island to be entered into rate equations 
(there is a different sign between terms and a balance between going to and from a charge state when calculating charging rates instead of a single-junction current) [10,12]. The $G$-function appearing here is

$$
G^{+-}=\left(1+G^{r} \sigma\right) g^{+-}\left(1+\sigma G^{a}\right)
$$

Then $G^{r}$ and $G^{a}$ have to be calculated in recursions in the same way as has been described for $T^{r}$ and $T^{a}$. Though subtle to realize, working with the $T$-functions has advantages. In (25) or the corresponding expression for the double junction $[10,17]$, a consistent choice of the $\omega$-zero reference level is crucial, which is more easily ensured with, for example, a $g_{R R}(\omega)$ in each term of the $T$-formula. Furthermore in a calculation for the double junction-with according for the fact that each hopping changes the state of the island by one charge-bare $\sigma$ at the ends of products would demand individual limits on calculations with $e$-and $h$-components at some stage. If $n$ denotes the maximum island charge considered, single hoppings that change to $n-1$ have to be taken with, whereas such that go to $n+1$ must not, because multiple reflection corrections for processes involving this state are missing. By the necessarily finite ranges for the $k$ - and $p$-indices on $T$, we limit the number of charges having gone through each junction from infinitely long ago up to any time and therefore cut processes exceeding these amounts. However, within these absolute charge limits (holes cancel electrons in the charge sums) all multiple back — and forth—and Andreev reflections are included.

\section{Conclusions and outlook}

It has been discussed in general how two perturbations, that is potentials or interactions, can be built into the system's Green's function successively. Looking at the relation of the total system's Green's function to that of the background system as a model, we called the transfer Green's function the quantity that relates analogously to the self-energy or bare interaction. For this transfer Green's function the second-step Dyson equation, which is of non-trivial structure, has been derived.

As an application we have presented the extension of an algorithm to calculate the transfer Green's function from a single quantum-point junction between superconducting bulk reservoirs to a series of two such contacts. The purpose of this study was to illustrate how effects from both interactions, the couplings across each junction in this case, individually or mixed together appear in the Green's function. The latter is an image of the interaction processesmultiple reflection here-occurring in the system. The Green's functions are an ingredient needed to calculate the current through the double-junction system. However, extending the single-junction current expression, which for the double junction provides charging rates of the island, to the case of coherent interaction over both junctions is a different issue. A representation in analogy to the single-junction current formula with mere two-products of transfer Green's functions [10] facilitates the physical interpretation in terms of transport processes as well as achieving numerical stability in modeling conduction properties. This derivation will be given elsewhere [17].

\section{Appendix}

As this may be quite instructive, we shall discuss here why even elaborate guesses for the second-step transfer Green's function's Dyson equation fail. To this end we use series expansions which generally are for $G$ and $V$ :

$$
G=g \sum_{n=0}^{\infty}(v g)^{n} \quad \text { and } \quad V=v \sum_{n=0}^{\infty}(g v)^{n} .
$$


Directly setting up the results for the system with two kinds of interaction we get

$$
G_{2}=g \sum_{n=0}^{\infty}\left(\left(v_{1}+v_{2}\right) g\right)^{n} \quad \text { and } \quad V_{2}=\left(v_{1}+v_{2}\right) \sum_{n=0}^{\infty}\left(g\left(v_{1}+v_{2}\right)\right)^{n} .
$$

With always $g$ in between and for $G$ also as outermost multipliers, every possible product of factors $v_{1}$ and $v_{2}$-that is every sequence-has to be contained exactly once. (Multiplication is not commutative here.) The right-hand sides of (9) and (16) reproduce the required sums, although the grouping of contributions may be a little confusing. We list categories of terms in the following. For the correct results these are complete and mutually exclusive. For the incorrect guesses of expansions for $V_{2}$ we comment where the mistakes are. (All sums over $n$ and $m$ run from 0 to $\infty$.)

Correct formula:

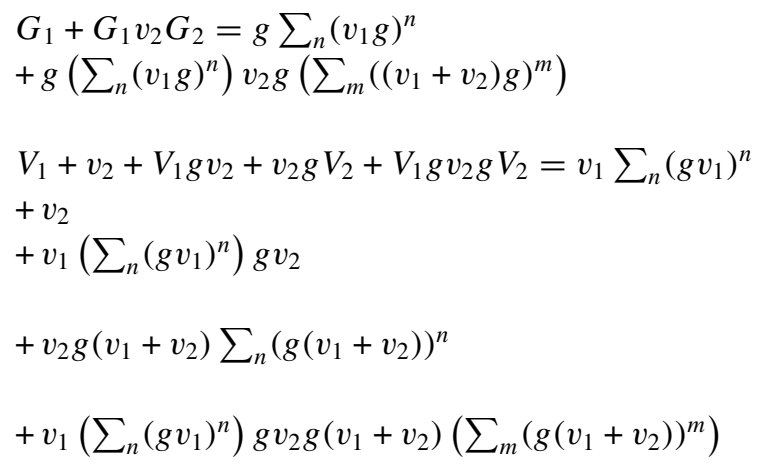

-with $v_{1}$ only

-all possible with $v_{1}$ and $v_{2}$

but at least containing one $v_{2}$

-with $v_{1}$ only

$-v_{2}$ just once

-with $v_{1}$ only (at least once)

but once $v_{2}$ at end

-at least two factors $v$

with $v_{2}$ at beginning

-at least three factors $v$

with $v_{1}$ at beginning

at least containing one $v_{2}$;

if end is $v_{2}$ : contains at least two $v_{2}$

Incorrect guesses:

$$
\begin{aligned}
& v_{2}+v_{2} g V_{2}= \\
& v_{2}+v_{2} g\left(v_{1}+v_{2}\right) \sum_{n}\left(g\left(v_{1}+v_{2}\right)\right)^{n} \quad \text {-terms beginning with } v_{1} \text { are missing } \\
& V_{1}+v_{2}+\left(V_{1}+v_{2}\right) g V_{2}= \\
& v_{1} \sum_{n}\left(g v_{1}\right)^{n} \quad \text {-with } v_{1} \text { only } \\
& +v_{2} \quad-v_{2} \text { just once } \\
& +\left[v_{1}\left(\sum_{n}\left(g v_{1}\right)^{n}\right)+v_{2}\right] g\left(v_{1}+v_{2}\right)\left(\sum_{m}\left(g\left(v_{1}+v_{2}\right)^{m}\right) \quad \text {-also contains products with } v_{1}\right. \text { only } \\
& \text { even multiple appearances } \\
& \text { of same power } \\
& V_{1}+v_{2}+V_{1} g v_{2}+\left(V_{1}+v_{2}+V_{1} g v_{2}\right) g V_{2}= \\
& v_{1} \sum_{n}\left(g v_{1}\right)^{n} \quad \text {-with } v_{1} \text { only } \\
& +v_{2} \quad \text {-just once } v_{2} \\
& +v_{1}\left(\sum_{n}\left(g v_{1}\right)^{n}\right) g v_{2} \quad \text {-with } v_{1} \text { only but once } v_{2} \text { at end } \\
& +\left[v_{1}\left(\sum_{n}\left(g v_{1}\right)^{n}\right)+v_{2}+v_{1}\left(\sum_{n}\left(g v_{1}\right)^{n}\right) g v_{2}\right] . \quad \text {-also contains terms with } v_{1} \text { only; } \\
& g\left(v_{1}+v_{2}\right)\left(\sum_{m}\left(g\left(v_{1}+v_{2}\right)\right)^{m}\right) \quad \text { terms with only } v_{1} \text { but once } \\
& v_{2} \text { at end also appear again }
\end{aligned}
$$




\section{References}

[1] Ohtaka K 1993 Green's functions Encyclopedia of Applied Physics (New York: VCH) p 341

[2] Whitten R C and McCormick P T 1995 Am. J. Phys. 43541

Hu B Y-K 2001 Am. J. Phys. 691280

[3] Chicanne C, David T, Quidant R, Weeber J C, Lacroute Y, Bourillot E and Dereux A 2002 Phys. Rev. Lett. 88097402

Dereux A, Deveaux E, Weeber J C, Goudonnet J P and Girard C 2000 J. Microsc. 202320

Weeber J C, Dereux A, Girard C, Colas des Francs G, Krenn J R and Goudonnet J P 2000 Phys. Rev. E 627381

Dereux A, Girard C and Weeber J C J. Chem. Phys. 1127775

[4] Nolting W 2002 Grundkurs Theoretische Physik 7, Viel-Teilchen-Theorie 5th edn (Berlin: Springer)

[5] Economou E N 1979 Green's Functions in Quantum Physics (Berlin: Springer)

[6] Datta S 1995 Electronic Transport in Mesoscopic Systems (Cambridge: Cambridge University Press)

[7] Cuevas J C, Martin-Rodero A and Levy Yeyati A 1996 Phys. Rev. B 547366

[8] Cuevas J C and Fogelström M 2001 Phys. Rev. B 64104502

Wohltat S, Pauly F, Viljas J K, Cuevas J C and Schön G 2007 Phys. Rev. B 76075413

Heurich J, Cuevas J C, Wenzel W and Schön G 2002 Phys. Rev. Lett. 88256803

Cuevas J C, Heurich J, Martin-Rodero A, Levy-Yeyati A and Schön G 2002 Phys. Rev. Lett. 88157001

[9] Schröter U 2003 Eur. Phys. J. B 33297

[10] Schröter U and Scheer E 2007 Phys. Rev. B 76205104

[11] Schröter U 2008 Preprint 0802.3001

[12] Schröter U and Scheer E 2006 Phys. Rev. B 74245301

[13] Beenakker C W J 1992 Phys. Rev. Lett. 4612841

[14] Scheer E, Agrait N, Cuevas J C, Levy-Yeyati A, Ludoph B, Martin-Rodero A, Rubio-Bollinger G, van Ruitenbeek $\mathrm{J}$ and Urbina C 1998 Nature 394154

Scheer E, Joyes P, Esteve D, Urbina C and Devoret M H 1997 Phys. Rev. Lett. 783535

[15] van Wees B J, van Houten H, Beenakker C W J, Williamson J G, Kouwenhoven L P, van der Marel D and Foxon C T 1988 Phys. Rev. Lett. 60848

[16] Goffman M F, Cron R, Levy-Yeyati A, Joyez P, Devoret M H, Esteve D and Urbina C 2000 Phys. Rev. Lett. 85170

Hadley P, Devigne E, Visscher E H, Läteenmäki S and Mooij J E 1998 Phys. Rev. B 5815317

[17] Schröter U and Scheer E 2008 J. Phys. A: Math. Theor. (submitted) 